\title{
TMEM140 is associated with the prognosis of glioma by promoting cell viability and invasion
}

\author{
Bin $\mathrm{Li}^{1 \dagger}$, Ming-Zhu Huang ${ }^{2 \dagger}$, Xiao-Qiang Wang ${ }^{1 *}$, Bang-Bao Tao ${ }^{1}$, Jun Zhong ${ }^{1}$, Xu-Hui Wang ${ }^{1}$, \\ Wen-Chuan Zhang ${ }^{1}$ and Shi-Ting $\mathrm{Li}^{1^{*}}$
}

\begin{abstract}
Background: Gliomas are the most common types of primary brain tumors in the adult central nervous system. TMEM140 is identified as an amplified gene in the human gastric cancer genome. However, the function of TMEM140 in gliomas has not been thoroughly elucidated. The aim of the current study was to determine the clinical significance of TMEM140 expression in patients with gliomas and its effect on tumor cell malignant phenotypes.

Methods: Immunohistochemical analysis and real-time reverse transcription PCR were performed to detect the expression levels of TMEM140 in 70 glioma brain tissue samples. Next, the correlation between the TMEM140 expression levels and the clinical characteristics and outcomes of glioma patients was statistically analyzed. TMEM140 expression was inhibited in two glioma cell lines (i.e., U87 and U373) using a knockdown method with small interfering RNA. Cell Counting Kit-8 and Transwell assays were used to investigate TMEM140 function during cell proliferation, invasion, and migration, respectively. Using flow cytometry and Western blot analysis, we subsequently determined the cell cycle and apoptosis profile of the TMEM140-silenced cells.

Results: TMEM140 protein expression was significantly higher in gliomas than in normal brain tissues $(p<0.0001)$. TMEM140 overexpression was strongly correlated with tumor size, histologic grade, and overall survival time $(P<0.05)$. TMEM140 decreased cell viability in vitro and dramatically decreased tumor volume in vivo. This phenomenon might be caused by G1 phase cell cycle arrest and cell apoptosis. TMEM140 silencing could suppress the viability, migration, and invasion of glioma cells.
\end{abstract}

Conclusions: Our results suggest that TMEM140 expression is a prognostic factor that might play an important role in the viability, migration, and invasion of glioma cells. This study highlights the importance of TMEM140 as a novel prognostic marker and as an attractive therapeutic target for gliomas.

Keywords: TMEM140, Glioma, Cell viability, Invasion, Prognosis

\section{Background}

Gliomas, which arise from glia cells, are the most common type of primary tumors in the central nervous system. Malignant glioma is classified as grade II-IV according to the 2007 World Health Organization (WHO) classification system [1]. Malignant glioma is a leading cause of death in patients, accounting for $45-55 \%$ of primary intracranial tumors. The annual incidence of malignant glioma is greater than 5 per 100,000 population [2]. Grade IV glioblastoma (GBM) is the most common and

\footnotetext{
*Correspondence: wangxq10@126.com; lishiting12@126.com

${ }^{\dagger}$ Equal contributors

'Department of Neurosurgery, Xinhua Hospital, Shanghai Jiaotong University School of Medicine, Shanghai 200092, China

Full list of author information is available at the end of the article
}

biologically aggressive malignant glioma. It is characterized by the hallmark features of unrestrained cellular proliferation, strong resistance to apoptosis, diffuse infiltration, vigorous angiogenesis and widespread genomic instability. With advances in aggressive surgery, radiation, and chemotherapy, the clinic survival rate for glioma has been greatly improved [3-5]. However, the median survival rate of patients with GBM remains less than 1 year [6, 7]. Understanding the molecular mechanisms involved in the formation and development of glioma is important for preventing and treating glioma and improving patient survival. Numerous molecular abnormalities, such as gene sequence alterations, DNA copy-number changes, chromosomal rearrangements, DNA methylation, and abnormal regulation

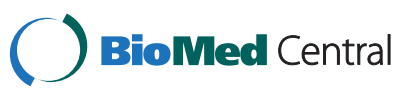

(c) 2015 Li et al. This is an Open Access article distributed under the terms of the Creative Commons Attribution License (http://creativecommons.org/licenses/by/4.0), which permits unrestricted use, distribution, and reproduction in any medium, provided the original work is properly credited. The Creative Commons Public Domain Dedication waiver (http:// creativecommons.org/publicdomain/zero/1.0/) applies to the data made available in this article, unless otherwise stated. 
of signaling pathways, have been associated with the tumorigenesis and development of glioma [8]. Inactivation of the $\mathrm{Rb}$ and $\mathrm{p} 53$ pathway [9-11], activation of receptor tyrosine kinases (RTKs) [8], and EGFRvIII activity [12], which are involved in tumor cell adhesion, migration, invasion, proliferation, and apoptosis, are observed in glioma [13-16]. However, the exact mechanism associated with this relationship remains unclear [15].

Transmembrane protein 140 (TMEM140, also known as FLJ11000) is a 185 amino acid protein encoded by a gene that maps to chromosome 7q33. Few studies have investigated the functions of this protein. Guan et al. reported an inhibitory effect of TMEM140 on herpes simplex virus 1 (HSV-1) proliferation [17]. Although TMEM140 has been identified as an amplified gene in the human gastric cancer genome [18], to the best of our knowledge, there have been no reports describing the functions of TMEM140 in tumorigenesis and development of tumors. The present study demonstrated the overexpression of TMEM140 in glioma tissues and analyzed the prognostic significance of TMEM140 expression in a large number of patients with gliomas. Next, we then investigated the effects of TMEM140 knockdown on cell viability and invasion in vitro and in vivo. Furthermore, we explored the underlying mechanisms. Our data suggest that TMEM140 can be a novel prognostic factor and potential treatment target for gliomas.

\section{Results}

Overexpression of TMEM140 and its prognostic significance in glioma patients

To investigate the protein expression profile of TMEM140 in gliomas, immunohistochemistry (IHC) was used in 70 formalin-fixed, paraffin-embedded tissue sections and 14 non-neoplastic brain tissues. As shown in Fig. 1a, TMEM140 can be observed in $67.1 \%$ (47/70) of the glioma specimens. Note that the control brain tissues had lower expression levels of TMEM140 protein compared with the glioma tissues.

We quantified the expression level of TMEM140 in glioma tissues compared with the control brain tissues (60 gliomas and 14 controls) using real-time reverse transcription PCR (RT-PCR) analysis. As shown in Fig. 1b, the level of TMEM140 in the tumor tissues was significantly higher than that in the controlled brain tissues ( $2.21 \pm 0.11$ vs. $0.87 \pm 0.07$, respectively, $P<0.0001)$. Next, we reanalyzed the high throughput RNA-sequencing data from the GBM cohort of the Cancer Genome Atlas

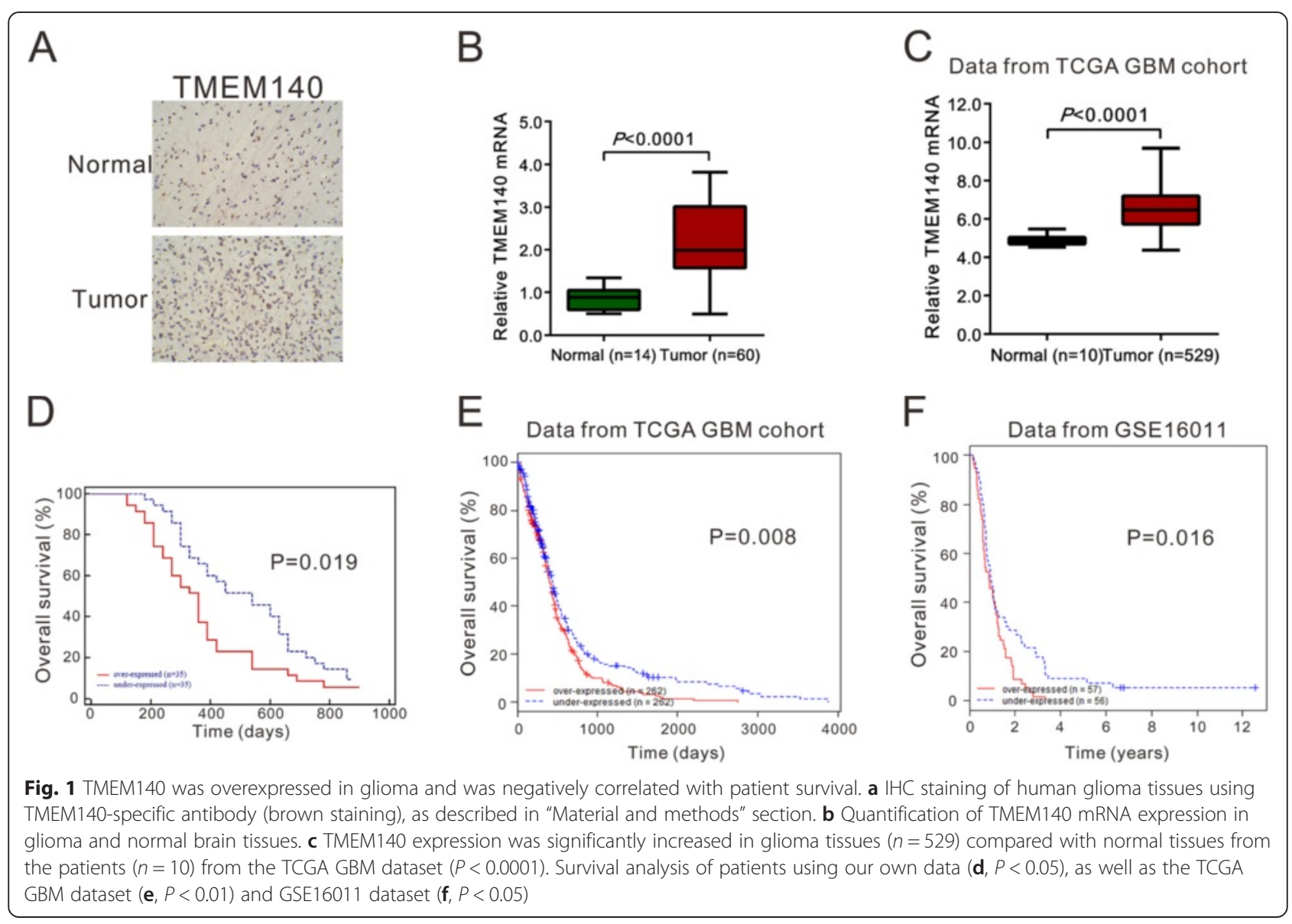


(TCGA), and we found that TMEM140 expression was significantly increased in glioma tissue compared with normal brain tissue (Fig. 1c, $P<0.001$ ).

The relationship between the clinicopathologic features and TMEM140 protein expression levels in 70 glioma patients is summarized in Table 1. A chi-square test showed that the increased expression level of TMEM140 was significantly correlated with tumor size $(P=0.0211)$ and pathological grade $(P=0.0108)$. As shown in Table 1 , there was no correlation between the expression level of TMEM140 protein and patient age or gender.

Therefore, we analyzed the relationship between TMEM140 protein overexpression and patient prognosis. A Kaplan-Meier survival curve was used to analyze the prognostic significance of TMEM140 expression. TMEM140 expression was divided into high-expression and low-expression groups. It was observed that patients with gliomas expressing high levels of TMEM140 sho wed statistically poorer prognoses compared with patients with gliomas expressing low levels of TMEM140$(P=0.019$; Fig. 1d). Furthermore, analyses of the TCGA GBM (https://tcga-data.nci.nih.gov/tcga/tcgaCancerDetails. jsp?diseaseType $=$ GBM\&diseaseName $=$ Glioblastoma $\% 20$ multiforme) and GSE16011 datasets [18] (http://www. ebi.ac.uk/arrayexpress/experiments/E-GEOD-16011/?query= GSE16011) also showed that patients with high TMEM140 protein expression had a significantly shorter overall survival rate time (TCGA, $P=0.008$; GSE16011, $P=0.016$; Fig. 1e, f).

\section{TMEM140 silencing suppressed the growth of glioma cells in vitro and in vivo}

To address the efficacy of TMEM140 on glioma cells, we knocked down TMEM140 in the glioma cell lines. We evaluated the expression levels of TMEM140 in five glioma cell lines (i.e., U87, U251, SHG44, U373, and T98G) through RT-PCR and Western blot analysis (Fig. 2a). Two cell lines (U87 and U373) that showed

Table 1 Correlation of TMEM140 expression in human glioma patients with different clinicopathological features $(n=70)$

\begin{tabular}{|c|c|c|c|c|}
\hline \multirow[t]{2}{*}{ Parameters } & \multirow[t]{2}{*}{ Characteristic } & \multicolumn{2}{|l|}{ TMEM140 } & \multirow[t]{2}{*}{$P$ value } \\
\hline & & High $(n=47)$ & Low $(n=23)$ & \\
\hline \multirow[t]{2}{*}{ Age (years) } & $\geq 55$ & 27 & 13 & \multirow[t]{2}{*}{0.6164} \\
\hline & $<55$ & 18 & 12 & \\
\hline \multirow[t]{2}{*}{ Gender } & Male & 16 & 10 & \multirow[t]{2}{*}{0.5990} \\
\hline & Female & 31 & 13 & \\
\hline \multirow[t]{2}{*}{ Tumor size } & $\geq 4.5 \mathrm{~cm}$ & 20 & 17 & \multirow[t]{2}{*}{$0.0211^{*}$} \\
\hline & $<4.5 \mathrm{~cm}$ & 27 & 6 & \\
\hline \multirow[t]{2}{*}{ WHO grade } & $|/| \mid$ & 15 & 15 & \multirow[t]{2}{*}{$0.0108^{*}$} \\
\hline & III/IV & 32 & 8 & \\
\hline
\end{tabular}

WHO World Health Organization

${ }^{*} P<0.05$ higher TMEM140 mRNA and protein expression levels were selected for RNA interference experiments. As shown in Fig. 2b, c, all three TMEM140 small interfering RNA (siRNA) sequences showed efficient silencing of TMEM140 expression based on Western blot and RTPCR analyses. TMEM140-RNAi-2 was the most effective protein, and it was used for the following assays.

Cell growth analysis was performed for two glioma cell lines (U87 and U373) transfected with TMEM140RNAi-2, which were then subjected to cell growth analysis. In a cell viability assay, both glioma cell lines showed significant reductions in cell viability through TMEM140 silencing compared with normal control cells (Fig. 3a, b). Seventy-two hours after siRNA transfection, cell viability was reduced 42.3 and $36.8 \%$ in the U87 and U373 cells, respectively.

We also found that the in vivo tumor growth and weight in the TMEM140-RNAi group were strongly inhibited compared with the normal control group (Fig. 3c, d). At the end point, the tumor volumes were $498.4 \pm 62.0$ and $1145.1 \pm 145.4 \mathrm{~mm}^{3}$ in the TMEM140RNAi and normal control groups, respectively $(P<0.01)$. Furthermore, the tumor weights were $0.44 \pm 0.04$ and $0.24 \pm 0.04 \mathrm{~g}$ in the TMEM140-RNAi and normal control groups, respectively $(P<0.01)$. Therefore, these findings showed that TMEM140 was required for the growth of glioma cells both in vitro and in vivo.

To address the mechanism underlying the growth suppression of glioma cells by TMEM140 knockdown, we then determined the cell cycle profile (Fig. 3e) of the TMEM140-silenced cells through flow cytometry. The results showed that TMEM140 silencing increased the population of the U87 and U373 cells in the G0/G1 phase by 53.5 and $43.3 \%$, respectively $(P<0.001)$.

TMEM140 silencing caused apoptosis in the glioma cells To probe the TMEM140-associated pathways in an unbiased manner, we performed Gene Set Enrichment Analysis (GSEA) based on the TCGA GBM dataset. We found that the KEGG apoptosis pathway (Fig. 4a) and cell adhesion molecules (Fig. 5a) were identified with the strongest association with higher TMEM40 expression levels.

The effect of TMEM140 on apoptosis in glioma cells (Fig. 4b) was investigated. TMEM140 knockdown in the glioma cells induced cell apoptosis at approximately 12 fold the rate in the corresponding normal control cells. Moreover, three apoptotic regulation factors [19] (i.e., $\mathrm{Bcl} 2, \mathrm{Bax}$, and cleaved caspase3) were evaluated using Western blot analysis. TMEM140 deficiency caused the up-regulation of the proteins promoting apoptosis (Bax and cleaved caspase 3 ) and the down-regulation of the protein inhibiting apoptosis (Bcl2) (Fig. 4c, d). 


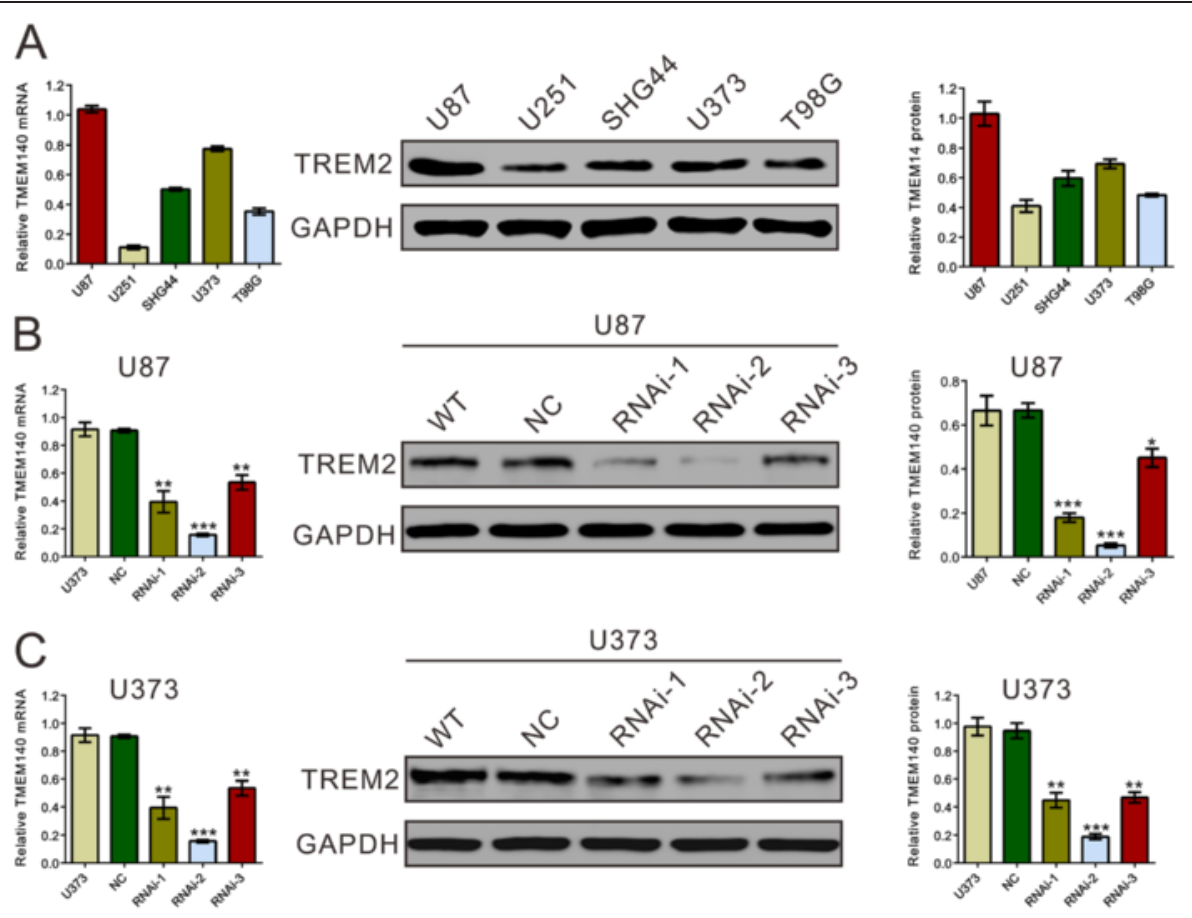

Fig. 2 Suppressing of TMEM140 expression by RNAi. a TMEM140 expression level in five glioma cell lines was analyzed by RT-PCR (left) and immunoblot (middle and right). b, c The effect of TMEM140 knockdown through siRNA silencing. The cells were transfected with normal control or TMEM140-RNAi for $48 \mathrm{~h}$ and then subjected to RT-PCR (left) and immunoblot analysis (middle and right) of the TMEM140 expression level. The representative images for immunoblot are shown in the middle panel, and data from three independent experiments were expressed as the mean \pm S.D. (right panel). Wild type: wild-type cells; normal control: scrambled siRNA transfected cells; RNAi-1, RNAi-2, and RNAi-3: TMEM140-RNAi-1, -2, and -3 transfected cells $\left({ }^{*} P<0.05,{ }^{* *} P<0.01,{ }^{* *} P<0.001\right.$ compared with normal control)

TMEM140 silencing diminished the adhesion, migration and invasion of glioma cells

Next, we next investigated the effects of TMEM140 on cell adhesion, migration, and invasion. TMEM140 knockdown decreased the adherent U87 and U373 cells by 61.5 and $43.3 \%$, respectively (Fig. 4b). TMEM140 knockdown strongly inhibited cell motility, with fewer than $60 \%$ of the U87 and U373 cells migrating (Fig. 4c). In contrast to the normal control cells, the invasive ability of the TMEM140 knockdown cells was greatly reduced (Fig. 4d). The number of invaded U87 and U373 cells with TMEM140 silenced was $35.9 \%$ compared with $42.9 \%$ for the normal control cells.

In contrast with the normal control cells, knockdown TMEM140 caused significantly down-regulated cell adhesion molecules (ICAM1, VCAM1, and Syndecan1) in the U87 and U373 cells. This finding is also consistent with functional characterization in vitro.

\section{Discussion}

TMEM140 gene is located on chromosome 7, and alterations of chromosome 7 are closely related to various cancers [20-25], such as breast cancer, prostate cancer, and gliomas. It remains unclear whether TMEM140 has important biological functions in tumors. In the present study, we found that TMEM140 was frequently overexpressed in $67.1 \%$ (47/70) of the glioma tissues. We hypothesized that the overexpression of TMEM140 may promote tumor cell growth in gliomas. To test this hypothesis, we examined the expression status of TMEM140 and the clinicopathologic features, as well as the biological significance of its expression in glioma cell lines. Consequently, the results implied that large tumor size, high histologic grade, and low patient survival rates were associated with high TMEM140 expression levels. Moreover, down-regulation of TMEM140 expression suppressed cell proliferation, migration, and invasion in two glioma cell lines (U87 and U373), which indicated that TMEM140 could be a potential therapeutic target of gliomas and warrants further investigation.

Unstrained cell proliferation is one of the most remarkable features of malignancies. Abnormal regulation in cell cycle and cell apoptosis frequently results in aberrant cell proliferation in cancer [26-29]. Here, the silencing of TMEM140 by siRNA transfection significantly induced G1-phase arrest (Fig. 3e) and cell apoptosis (Fig. 4), which may trigger the inhibitory effects of TMEM140 siRNA on cell proliferation in vitro and in vivo.

Cell adhesion, migration, and invasion are key steps for tumorigenicity in tumor progression [26, 30-32]. Based on GSEA of the TCGA GBM dataset, we found that 


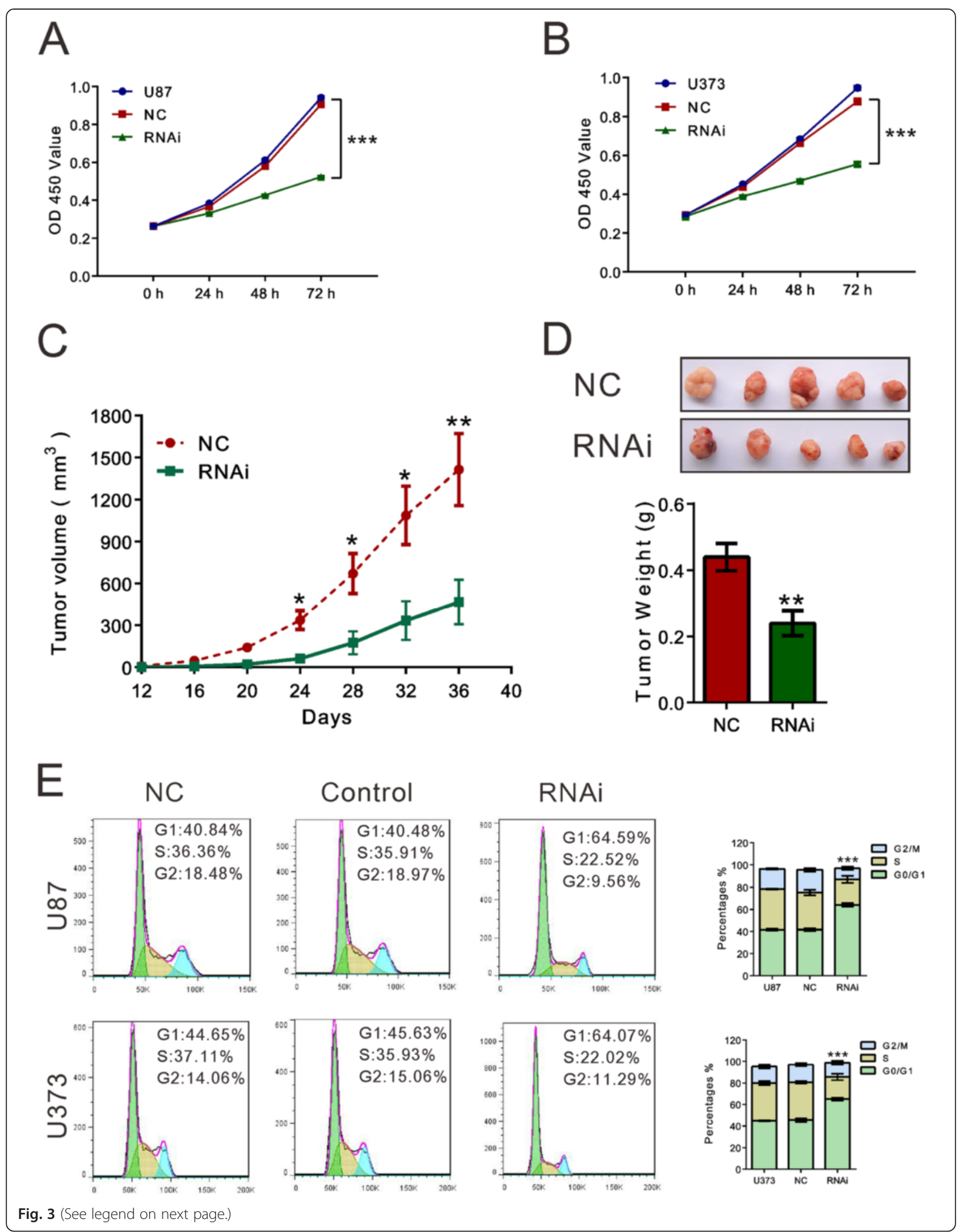


(See figure on previous page.)

Fig. 3 The growth-suppressive effect of TMEM140 silencing on GBM cells in vitro and in vivo. $\mathbf{a}$, b The cells were transfected with TMEM140-RNAi-2 or normal control and then subjected to cell proliferation assay by CCK-8. TMEM140 knockdown via siRNA silencing inhibited the proliferation of U87 (a) and U373 (b) cells. c, d TMEM140 knockdown inhibited the growth of U87 cells in vivo. The representative images for xenograft tumor on the nude mouse are shown in Fig. 1d (top panel), and the tumor growth curve or tumor weight is shown in Fig. 1c, $d(n=5)$. e U87 and U373 cells were transfected with the indicated siRNA. Cells were collected after $48 \mathrm{~h}$, and cell cycle profile was analyzed using flow cytometry. The representative images are shown in the left panel, and data from three independent experiments are expressed as the mean \pm S.D. (right panel) $\left({ }^{*} P<0.05\right.$, ${ }^{* *} P<0.01$, ***P $<0.001$ compared with normal controls)

TMEM140 was associated with cellular adhesion molecules (Fig. 5a), which have been implicated in tumor progression. In addition, our in vitro data demonstrated that the depletion of TMEM140 significantly suppressed cell adhesion, migration, and invasion (Fig. 5b-d). Furthermore, TMEM140 knockdown remarkably decreased the protein levels of ICAM1, VCAM1, and Syndecan1 (Figs. 5e, f), which are considered to be important in glioma cell migration and invasion [33]. These data provided useful information explaining how TMEM140 siRNA inhibited glioma cell migration and invasion, although more in-depth studies are necessary.

\section{Conclusion}

In conclusion, we found for the first time that TMEM140 accumulates in gliomas. The higher level of TMEM140 is strongly correlated with tumor size, histologic grade, and overall survival time in this disease. TMEM140 silencing could suppress the viability, migration, and invasion of glioma cells. Our data demonstrated that TMEM140 is not only a prognostic biomarker but also a therapeutic target in human gliomas.

\section{Materials and methods}

\section{Tissue collection}

A total of 70 patients with glioma were enrolled in this study. The patients underwent surgical removal of their tumors at Xinhua Hospital, Shanghai Jiaotong University School of Medicine, Shanghai, China, from January 2009 to December 2010. All patients had intracranial gliomas and no history of other malignancies. Histological sections of the primary resected surgical specimens were

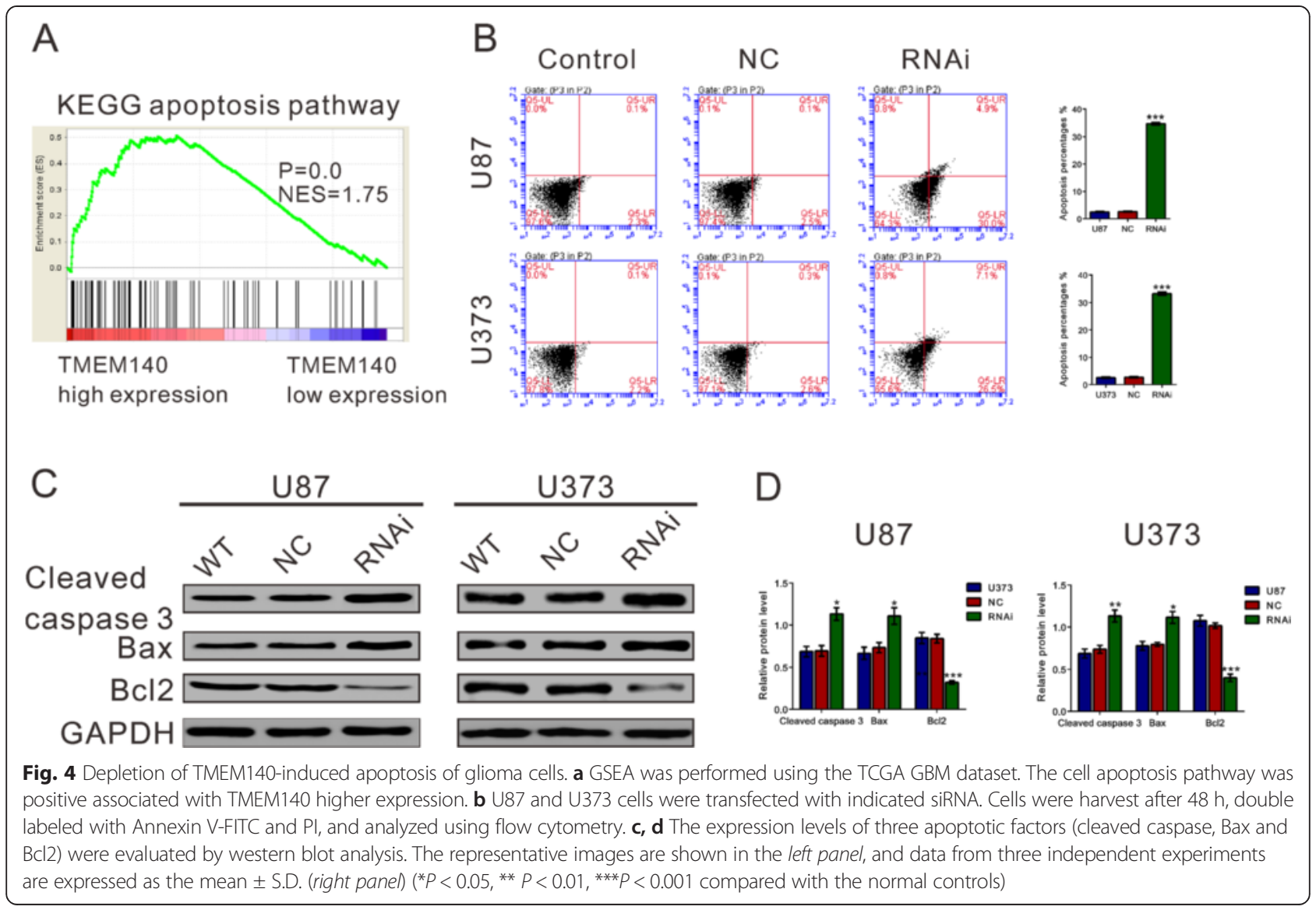


A

KEGG cell adhesion molecules

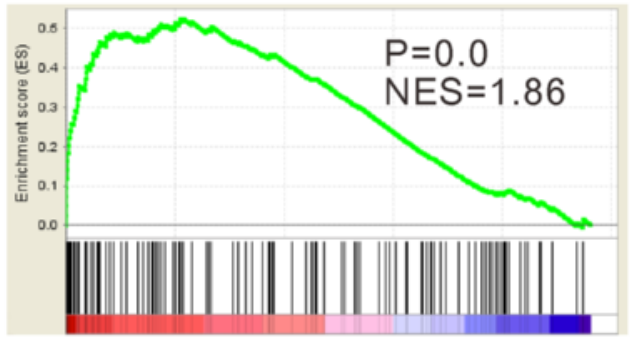

TMEM140 TMEM140

high expression low expression

C
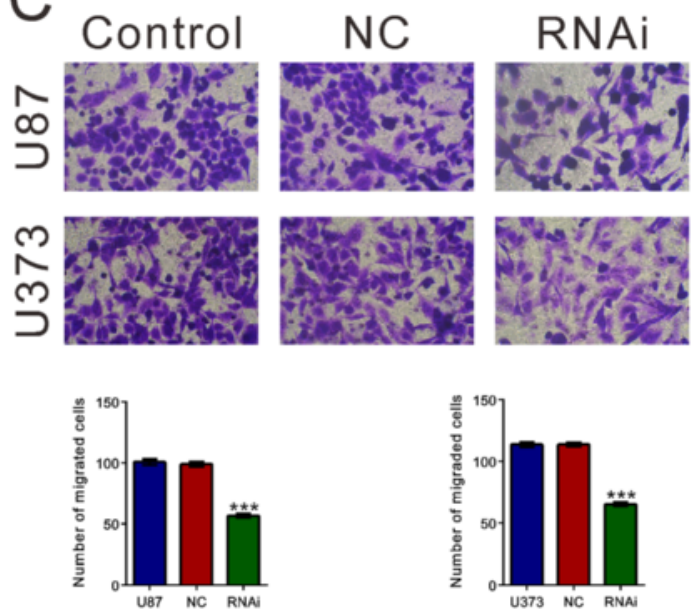

E

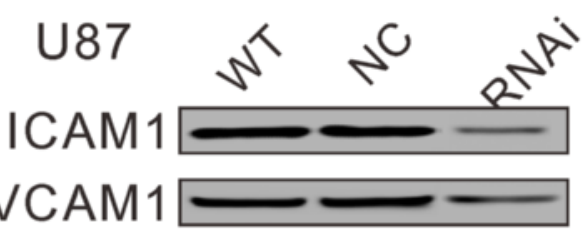

Syndecan 1

GAPDH
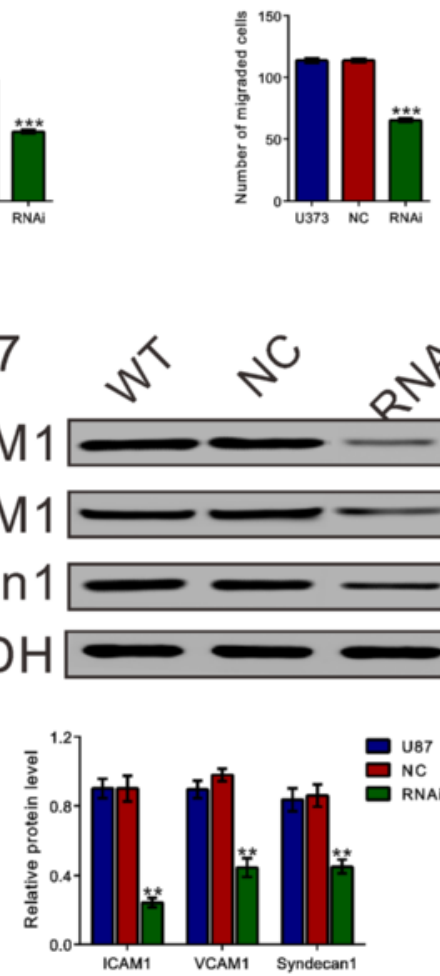

Fig. 5 (See legend on next page.)
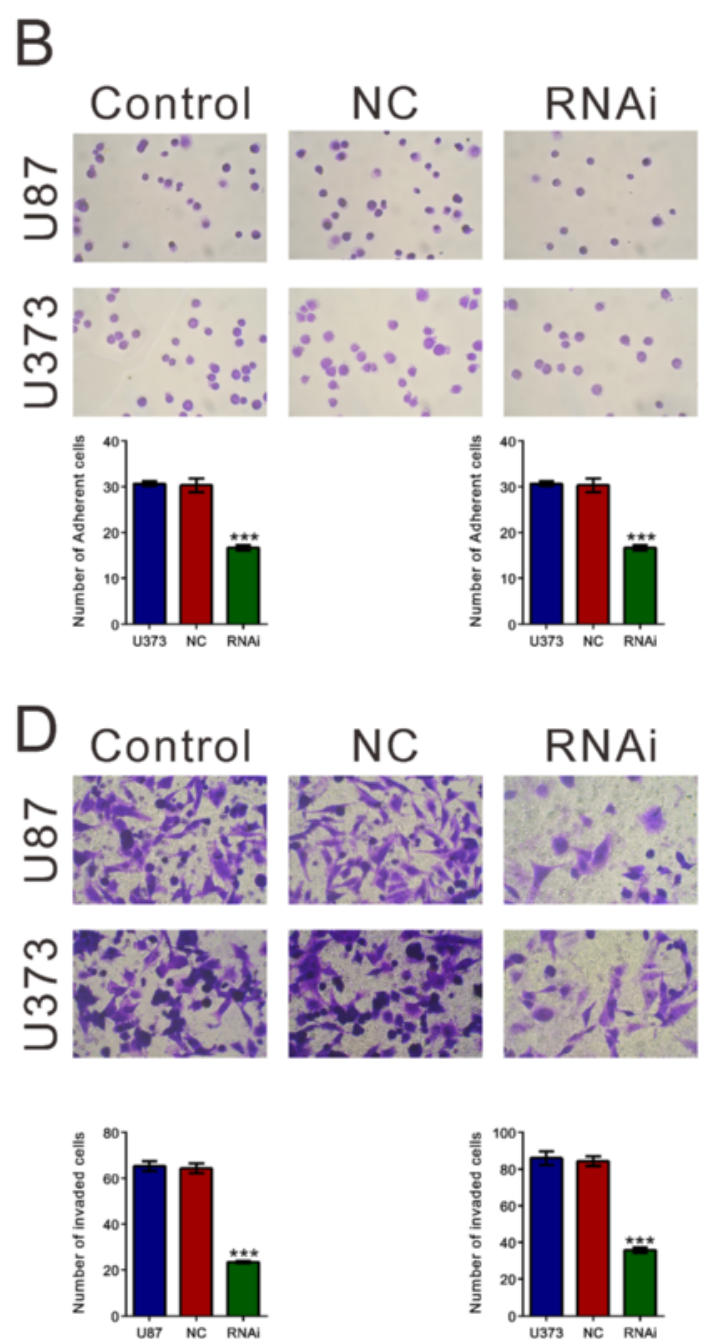

F
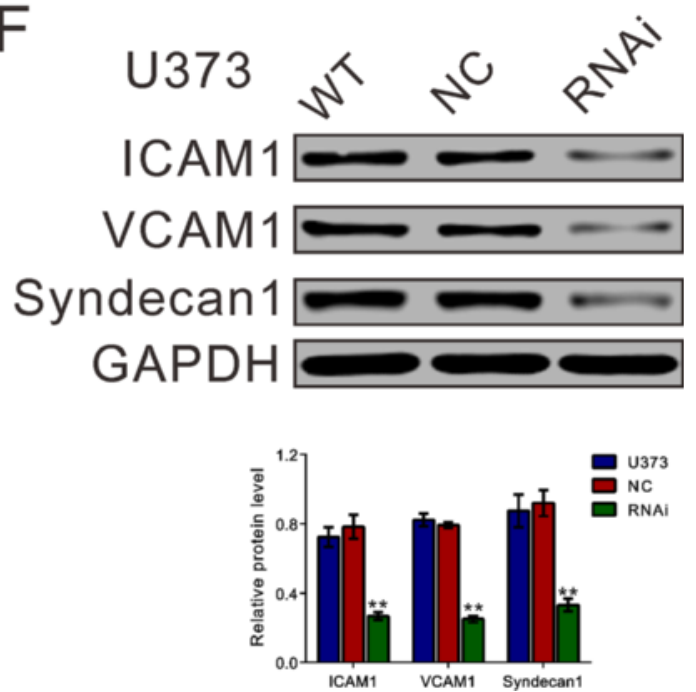
(See figure on previous page.)

Fig. 5 TMEM140 silencing suppressed adhesion, migration, and invasion in the glioma cells. a Based on the TCGA GBM dataset, GSEA showed that KEGG cell adhesion molecules were positively associated with higher TMEM140 expression levels. The cells were transfected with normal control or TMEM140-RNAi for $48 \mathrm{~h}$ and subjected to cell adhesion, migration, and invasion assay. b TMEM140 knockdown inhibited glioma cell adhesion. c TMEM140 knockdown inhibited the migration abilities of glioma cells. d TMEM140 knockdown suppressed the invasive abilities of the glioma cells. The representative images are shown in the upper panel, and data from three independent experiments are expressed as the mean \pm S.D. e, $\mathbf{f}$ Expression levels of cell adhesion molecules, ICAM1, VCAM1, and Syndecan1 were evaluated by Western blot analysis (Iower panel). $\left({ }^{*} P<0.05\right.$, ${ }^{* *} P<0.01,{ }^{* *} P<0.001$ compared with the normal controls)

reviewed by authoritative pathologists, according to the 2007 WHO Classification of Tumors of the Central Nervous System [1]. The patients' clinical characteristics, such as age, gender, and tumor size, were collected for statistical analysis. All patients had complete 5-year follow-ups until death or until the last follow-up. Overall survival time was calculated from the date of the initial surgical operation until death. Additionally, 14 non-neoplastic brain tissue samples were obtained from surgical procedures for epilepsy. The study protocol was approved by the local, independent ethics committee at Xinhua Hospital, Shanghai Jiaotong University School of Medicine. Written informed consent was obtained from all patients.

\section{Cell culture}

The human glioblastoma cell lines (U87, U251, SHG44, U373, and T98G) were purchased from the Cell Bank of the Shanghai Branch of the Chinese Academy of Sciences (Shanghai, China). All culture media were supplemented with $10 \%$ fetal bovine serum (FBS, Hyclone, Logan, UT, USA) and $100 \mathrm{U} / \mathrm{mL}$ penicillin/streptomycin (Gibco, Carlsbad, CA, USA). U87, U373, and T98G were cultured in Eagle's Minimum Essential Medium (MEM, Hyclone), while the U251 and SHG44 cells were cultured in Dulbecco's Modified Eagle's medium (DMEM, Hyclone). All cell lines were maintained in a humidified atmosphere, with $5 \% \mathrm{CO}_{2}$ at $37^{\circ} \mathrm{C}$.

\section{Gene silencing}

Two glioma cell lines, U87 and U373, were transfected with siRNA oligonucleotides using Lipofectamine 2000 (Invitrogen, Carlsbad, CA, USA). Briefly, siRNA and Lipofectamine 2000 were incubated separately with OptiMEM for $5 \mathrm{~min}$ and mixed together for $20 \mathrm{~min}$ at room temperature, and then the mixture was applied to cells plated in $4 \mathrm{~mL}$ of medium (final concentration of siRNA, $60 \mathrm{nM}$ ). The siRNA sequences were as follows:

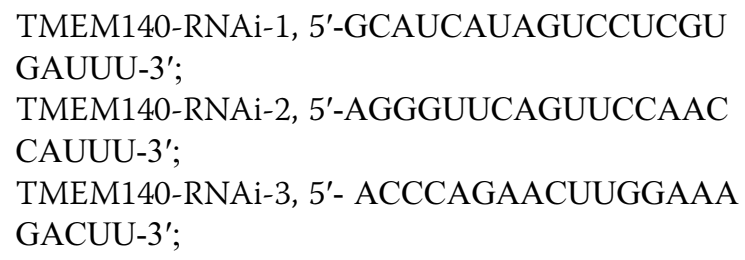

and normal control scrambled siRNA, 5'- UUGUAC UACACAAAAGUACUG-3'.

All siRNAs were purchased from GenePharma (Shanghai, China).

\section{Immunohistochemical analysis}

Tissues, $5-\mu \mathrm{m}$ sections, were dehydrated and subjected to antigen retrieval and endogenous peroxidase blocking. The sections were subsequently incubated with TMEM140-specific antibody (Abcam, Cambridge, MA, USA) overnight at $4{ }^{\circ} \mathrm{C}$ followed by incubated with goat anti-rabbit secondary antibody, and the sections were subsequently developed using 3,3-diaminobenzidine (DAB) solution and counterstained with hematoxylin. The staining assessments were performed by two senior neuropathologists who were blinded to the clinical parameters. The specimens were graded into two groups, according to the extent of positivity, as follows: low, $<25 \%$ of the tumor cells showed positive stain; and high, $>25 \%$ of tumor cells showed positive stain.

\section{Real-time reverse transcription PCR (RT-PCR)}

Total RNA was extracted from the glioma and normal brain tissues or from the cell lines using TRIzol reagent (Invitrogen), according to the manufacturer's instructions. DNase I-treated RNA was reverse transcribed using a first-strand cDNA synthesis kit (Fermentas, Hanover, MD, USA). Each cDNA was amplified using a standard SYBR Green kit (Thermo Fisher Scientific, Rockford, IL, USA) and then loaded into the 7300 RTPCR Detection System (Applied Biosystems, Foster City, CA, USA). The thermal cycling conditions were as follows: $95{ }^{\circ} \mathrm{C}$ for $10 \mathrm{~min}$ for the first step, and then for ensuing 40 cycles, $95{ }^{\circ} \mathrm{C}$ for $15 \mathrm{~s}$ and $60{ }^{\circ} \mathrm{C}$ for $45 \mathrm{~s}$. The following PCR primers were used:

TMEM140 (NM_018295.4), forward primer: 5'-AAACCATGCAGCTCATTGTC-3'; reverse primer: 5'-TCGTTTGTTGCCGTAAGTTC-3'; GAPDH (NM_001256799.2), forward primer: 5'-AATCCCATCACCATCTTC-3'; and reverse primer: 5'-AGGCTGTTGTCATACTTC-3'. 


\section{Western blot analysis}

Cell lysates were extracted with RIPA cell lysis buffer, and the protein concentration in the lysates was quantified using an enhanced bicinchoninic acid protein assay kit (Thermo Fisher Scientific). Protein samples $(30-50 \mu \mathrm{g}$ ) were loaded for immunoblotting using antibodies against TMEM140, Bcl2, Bax, intercellular adhesion molecule 1 (ICAM1), vascular cell adhesion molecule1 (VCAM1) and Syndecan1 (Abcam, Cambridge, MA, USA), and GAPDH (Cell Signaling Technology, Danvers, MA, USA). Specific proteins were detected with enhanced chemiluminescence (ECL, Millipore, Bredford, USA). Band density was measured (ImageJ software) and normalized to GAPDH.

\section{Tumor formation assay}

Five-week-old male athymic nude mice were purchased from the Shanghai Experimental Animal Center. U87 cells transfected with TMEM140-siRNA or the control siRNA for $24 \mathrm{~h}$ were trypsinized, resuspended in PBS, and then subcutaneously injected into the flank of nude mice with $2 \times 10^{6}$ cells per injection ( $n=5$ per group). Tumor size was measured with a Vernier caliper every 4 days and calculated as (length $\times$ width $\left.^{2}\right) / 2$. All procedures were performed in accordance with the National Institutes of Health Guide for the Care and Use of Laboratory Animals.

\section{Cell viability assay}

Cell viability was assayed using the Cell Counting Kit-8 (CCK-8, Dojindo Laboratories, Tokyo, Japan) according to the manufacturer's instructions. Briefly, treated and untreated cells were seeded in 96-well plates at a density of 1000-1500 cells per well and incubated for 1,2 , or 3 days. At the indicated time point, the CCK8 solution was added to each well and incubated for $1 \mathrm{~h}$. The absorbance value (optical density) of each well was measured at $450 \mathrm{~nm}$. For each experimental condition, three wells were used. All experiments were performed in triplicate.

\section{Evaluation of cell cycle distribution and cell apoptosis by flow cytometry}

Treated and untreated cells were harvested and fixed in $70 \%$ ethanol at $-20{ }^{\circ} \mathrm{C}$ overnight. Fixed cells were then washed in PBS and stained with $0.1 \mathrm{mg} / \mathrm{ml}$ propidium iodide (PI, Sigma, St. Louis, MO, USA) containing $1 \mathrm{mg} /$ $\mathrm{ml} \mathrm{RNase} \mathrm{A}$ for $30 \mathrm{~min}$ at $37^{\circ} \mathrm{C}$. Intensities of fluorescence signals were measured on a FACScan flow cytometer (BD Biosciences, San Jose, CA, USA). The percentages of cells in the G0/G1, S, and G2/M phases were determined using FlowJo software (Tree Star). All experiments were performed three times.

The percentage of cells actively undergoing apoptosis was determined by double staining with Annexin V-fluorescein isothiocyanate (FITC) and PI. Transfected cells were harvested, double-labeled with Annexin V-FITC and PI apoptosis detection kits (KeyGEN Biotech, Nanjing, China), and analyzed using a FACScan flow cytometry. At least 20,000 cells were acquired for each sample. The experiments were performed in triplicate.

\section{Cell adhesion assay}

Treated and untreated cells were seeded onto fibronectincoated 12-well plates at a density of $1 \times 10^{5}$ cells per well and allowed to adhere at $37{ }^{\circ} \mathrm{C}$ for $1 \mathrm{~h}$. After the nonadherent cells were washed with a PBS, the attached cells were fixed in $4 \%$ paraformaldehyde and stained with GIEMSA solution. The adherent cells were photographed and counted under an Olympus inverted microscope (Lake Success, NY, USA). The experiments were performed in triplicate.

\section{Cell migration and invasion assays}

In vitro cell migration and invasion assays were performed using 24-well Transwell chambers $(8-\mu \mathrm{m}$ pores, Coring Incorporated, NY, USA). The treated and untreated cells were cultured in the top chamber with serum-free media in triplicate at $5 \times 10^{4}$ cells per well. In the lower chamber, 500- $\mu$ l media with $10 \%$ FBS was added as a chemoattractant. After $24 \mathrm{~h}$ of cultivation, the media from the chamber and the Transwell were removed, and the chamber was gently wiped with a cotton swab. The migrated cells were fixed in $4 \%$ paraformaldehyde, stained with crystal violet solution and counted under a microscope in five fields $(\times 200)$. The procedure for the cell invasion assay was similar to the cell migration assay, except that the Transwell membranes were precoated with Matrigel (BD Biosciences).

\section{Gene Set Enrichment Analysis (GSEA)}

Glioblastoma multiforme (GBM) cohort downloaded from The Cancer Genome Atlas (TCGA) was analyzed by GSEA. GSEA was performed using GSEA software, Version 2.0.1, which was obtained from the Broad Institute (http://www.broad.mit.edu/gsea; ref. 20), as previously described [34-37]. Gene set permutations were performed 1000 times for each analysis. The nominal $P$ value and normalized enrichment score (NES) were used to sort the pathways enriched in each phenotype.

\section{Statistical analysis}

All statistical analyses were performed using the SPSS 16.0 software package. Data are presented as the mean \pm S.D. A two-tailed Student's $t$-test was used for between-group comparisons. The correlation between the IHC staining score and clinicopathologic variables was evaluated using a chi-squared test. Kaplan-Meier survival curves were calculated using death as the end point. The difference in the overall survival curves was examined with a log- 
rank test. Differences were considered to be statistically significant at $P<0.05$.

\section{Ethical approval}

All procedures performed in studies involving human participants were in accordance with the ethical standards of the local, independent ethics committee at Xinhua Hospital, Shanghai Jiaotong University School of Medicine and with the 1964 Helsinki declaration and its later amendments or comparable ethical standards. Written informed consent was obtained from all patients.

All procedures performed in studies involving animals were in accordance with the ethical standards of the local, independent ethics committee at Xinhua Hospital, Shanghai Jiaotong University School of Medicine.

\section{Ethics, consent, and permissions}

Informed consent was obtained from all individual participants included in the study.

\section{Consent to publish}

Authors have obtained consent to publish from the participant (or legal parent or guardian for children) to report individual patient data. This is required where an article reports an individual participant's data in any form (including images, videos, voice recordings, etc.).

\section{Competing interests}

The authors have obtained consent from the participants (or their legal parents or guardians) to report and publish individual patient data. This permission is required when an article reports an individual participant's data in any form (including images, videos, voice recordings, etc.).

\section{Authors' contributions}

$\mathrm{BL}$ contributed to the experiment design and implementation, manuscript draft, and data analysis. MZH contributed to the experiment implementation and data analysis. XQW conceived or designed the experiments. BBT and XHW performed the experiments. JZ and WCZ analyzed the data and wrote the manuscript. STL conceived or designed the experiments, performed the experiments, and wrote the manuscript. All authors read and approved the final manuscript.

\section{Acknowledgments}

The authors would like to thank the following people for their invaluable contribution: Miss Lin Zhu, for proofreading and editing of manuscript; Professor Jian-Kang Shen, for his valuable advices and discussions for this work; some patients and their guardians, for their supports in related examinations and follow-up. This work is supported by grants from China Postdoctoral Science Foundation (No. 20100480568).

\section{Author details}

${ }^{1}$ Department of Neurosurgery, Xinhua Hospital, Shanghai Jiaotong University School of Medicine, Shanghai 200092, China. ${ }^{2}$ Department of Oncology, Fudan University Shanghai Cancer Center, Shanghai 200032, China.

Received: 8 May 2015 Accepted: 8 July 2015

Published online: 22 July 2015

\section{References}

1. Louis DN, Ohgaki H, Wiestler OD, Cavenee WK, Burger PC, Jouvet A, et al. The 2007 WHO classification of tumours of the central nervous system. Acta Neuropathol. 2007;114(2):97-109. doi:10.1007/s00401-007-0243-4.
2. Ohgaki H. Epidemiology of brain tumors. Methods Mol Biol. 2009;472: 323-42. doi:10.1007/978-1-60327-492-0_14.

3. Stupp R, Mason WP, van den Bent MJ, Weller M, Fisher B, Taphoorn MJ, et al. Radiotherapy plus concomitant and adjuvant temozolomide for glioblastoma. N Engl J Med. 2005;352(10):987-96. doi:10.1056/NEJMoa043330.

4. Oermann E, Collins BT, Erickson KT, Yu X, Lei S, Suy S, et al. CyberKnife enhanced conventionally fractionated chemoradiation for high grade glioma in close proximity to critical structures. J Hematol Oncol. 2010;3:22. doi:10.1186/1756-8722-3-22.

5. Ottenhausen M, Krieg SM, Meyer B, Ringel F. Functional preoperative and intraoperative mapping and monitoring: increasing safety and efficacy in glioma surgery. Neurosurg Focus. 2015;38(1), E3. doi:10.3171/ 2014.10.FOCUS14611.

6. Jemal A, Siegel R, Xu J, Ward E. Cancer statistics, 2010. CA Cancer J Clin. 2010;60(5):277-300. doi:10.3322/caac.20073.

7. Davis FG, Freels S, Grutsch J, Barlas S, Brem S. Survival rates in patients with primary malignant brain tumors stratified by patient age and tumor histological type: an analysis based on Surveillance, Epidemiology, and End Results (SEER) data, 1973-1991. J Neurosurg. 1998;88(1):1-10. doi:10.3171/jns.1998.88.1.0001.

8. Huse JT, Holland EC. Targeting brain cancer: advances in the molecular pathology of malignant glioma and medulloblastoma. Nat Rev Cancer. 2010;10(5):319-31. doi:10.1038/nrc2818.

9. Ueki K, Ono Y, Henson JW, Efird JT, von Deimling A, Louis DN. CDKN2/p16 or RB alterations occur in the majority of glioblastomas and are inversely correlated. Cancer Res. 1996;56(1):150-3.

10. Schmidt EE, Ichimura K, Reifenberger G, Collins VP. CDKN2 (p16/MTS1) gene deletion or CDK4 amplification occurs in the majority of glioblastomas. Cancer Res. 1994;54(24):6321-4.

11. England B, Huang T, Karsy M. Current understanding of the role and targeting of tumor suppressor p53 in glioblastoma multiforme. Tumour biology : the journal of the International Society for Oncodevelopmental Biology and Medicine. 2013;34(4):2063-74. doi:10.1007/s13277-013-0871-3.

12. Shen CJ, Yang YX, Han EQ, Cao N, Wang YF, Wang Y, et al. Chimeric antigen receptor containing ICOS signaling domain mediates specific and efficient antitumor effect of T cells against EGFRvIll expressing glioma. J Hematol Oncol. 2013:6:33. doi:10.1186/1756-8722-6-33.

13. Hegi ME, Diserens AC, Gorlia T, Hamou MF, de Tribolet N, Weller M, et al. MGMT gene silencing and benefit from temozolomide in glioblastoma. N Engl J Med. 2005;352(10):997-1003. doi:10.1056/NEJMoa043331.

14. Maher EA, Furnari FB, Bachoo RM, Rowitch DH, Louis DN, Cavenee WK, et al. Malignant glioma: genetics and biology of a grave matter. Genes Dev. 2001;15(11):1311-33. doi:10.1101/gad.891601.

15. Cancer Genome Atlas Research N. Comprehensive genomic characterization defines human glioblastoma genes and core pathways. Nature. 2008:455(7216):1061-8. doi:10.1038/nature07385.

16. Yan H, Parsons DW, Jin G, McLendon R, Rasheed BA, Yuan W, et al. IDH1 and IDH2 mutations in gliomas. N Engl J Med. 2009;360(8):765-73. doi:10.1056/NEJMoa0808710.

17. Guan Y, Guo L, Yang E, Liao Y, Liu L, Che Y, et al. HSV-1 nucleocapsid egress mediated by UL31 in association with UL34 is impeded by cellular transmembrane protein 140. Virology. 2014;464-465:1-10. doi:10.1016/ j.virol.2014.06.034.

18. Yang S. Gene amplifications at chromosome 7 of the human gastric cancer genome. International journal of molecular medicine. 2007;20(2):225-31.

19. Hengartner MO. The biochemistry of apoptosis. Nature. 2000:407(6805):770-6.

20. Korc M, Meltzer P, Trent J. Enhanced expression of epidermal growth factor receptor correlates with alterations of chromosome 7 in human pancreatic cancer. Proc Natl Acad Sci U S A. 1986:83(14):5141-4.

21. Waldman FM, Carroll PR, Kerschmann R, Cohen MB, Field FG, Mayall BH. Centromeric copy number of chromosome 7 is strongly correlated with tumor grade and labeling index in human bladder cancer. Cancer Res. 1991;51(14):3807-13.

22. Alcaraz A, Takahashi S, Brown JA, Herath JF, Bergstralh EJ, Larson-Keller JJ, et al. Aneuploidy and aneusomy of chromosome 7 detected by fluorescence in situ hybridization are markers of poor prognosis in prostate cancer. Cancer Res. 1994;54(15):3998-4002.

23. Lee JS, Pathak S, Hopwood V, Tomasovic B, Mullins TD, Baker FL, et al. Involvement of chromosome 7 in primary lung tumor and nonmalignant normal lung tissue. Cancer Res. 1987;47(23):6349-52.

24. Beroukhim R, Getz G, Nghiemphu L, Barretina J, Hsueh T, Linhart D, et al. Assessing the significance of chromosomal aberrations in cancer: 
methodology and application to glioma. Proc Natl Acad Sci U S A. 2007;104(50):20007-12. doi:10.1073/pnas.0710052104.

25. Bieche I, Champeme MH, Matifas F, Hacene K, Callahan R, Lidereau R. Loss of heterozygosity on chromosome $7 \mathrm{q}$ and aggressive primary breast cancer. Lancet. 1992;339(8786):139-43.

26. Hanahan D, Weinberg RA. Hallmarks of cancer: the next generation. Cell. 2011;144(5):646-74. doi:10.1016/j.cell.2011.02.013.

27. Gravina GL, Senapedis W, McCauley D, Baloglu E, Shacham S, Festuccia C. Nucleo-cytoplasmic transport as a therapeutic target of cancer. J Hematol Oncol. 2014;7:85. doi:10.1186/s13045-014-0085-1.

28. Shen Q, Liu S, Chen Y, Yang L, Chen S, Wu X, et al. Proliferation inhibition and apoptosis induction of imatinib-resistant chronic myeloid leukemia cells via PPP2R5C down-regulation. J Hematol Oncol. 2013;6:64. doi:10.1186/ 1756-8722-6-64

29. Heller G, Rommer A, Steinleitner K, Etzler J, Hackl H, Heffeter P, et al. EVI1 promotes tumor growth via transcriptional repression of MS4A3.J Hematol Oncol. 2015;8:28. doi:10.1186/s13045-015-0124-6.

30. Parikh K, Cang S, Sekhri A, Liu D. Selective inhibitors of nuclear export (SINE) —a novel class of anti-cancer agents. J Hematol Oncol. 2014;7:78. doi:10.1186/s13045-014-0078-0

31. Andolfi L, Bourkoula E, Migliorini E, Palma A, Pucer A, Skrap M, et al. Investigation of adhesion and mechanical properties of human glioma cells by single cell force spectroscopy and atomic force microscopy. PLoS One. 2014;9(11), e112582. doi:10.1371/journal.pone.0112582.

32. Gu F, Wang L, He J, Liu X, Zhang H, Li W, et al. Girdin, an actin-binding protein, is critical for migration, adhesion, and invasion of human glioblastoma cells. J Neurochem. 2014;131(4):457-69. doi:10.1111/jnc.12831.

33. Demuth T, Berens ME. Molecular mechanisms of glioma cell migration and invasion. J Neurooncol. 2004;70(2):217-28. doi:10.1007/s11060-004-2751-6.

34. Kapoor A, Yao W, Ying H, Hua S, Liewen A, Wang Q, et al. Yap1 activation enables bypass of oncogenic Kras addiction in pancreatic cancer. Cell. 2014;158(1):185-97. doi:10.1016/j.cell.2014.06.003.

35. Yuan JH, Yang F, Wang F, Ma JZ, Guo YJ, Tao QF, et al. A long noncoding RNA activated by TGF-beta promotes the invasion-metastasis cascade in hepatocellular carcinoma. Cancer Cell. 2014;25(5):666-81. doi:10.1016/ j.ccr.2014.03.010

36. Prensner JR, lyer MK, Sahu A, Asangani IA, Cao Q, Patel L, et al. The long noncoding RNA SChLAP1 promotes aggressive prostate cancer and antagonizes the SWI/SNF complex. Nat Genet. 2013;45(11):1392-8. doi:10.1038/ng.2771

37. Schiaffino-Ortega S, Balinas C, Cuadros M, Medina PP. SWI/SNF proteins as targets in cancer therapy. J Hematol Oncol. 2014;7(1):81. doi:10.1186/s13045-014-0081-5.

\section{Submit your next manuscript to BioMed Central and take full advantage of:}

- Convenient online submission

- Thorough peer review

- No space constraints or color figure charges

- Immediate publication on acceptance

- Inclusion in PubMed, CAS, Scopus and Google Scholar

- Research which is freely available for redistribution 